\title{
Psychomotor reminiscence and the menstrual cycle
}

\author{
ELIZABETH LAMSON-McBRIDE and R. B. PAYNE \\ University of Georgia, Athens, Georgia 30602
}

\begin{abstract}
As in Wells and Payne (1979), psychomotor reminiscence was studied in relation to endogenous variations in estrogenic hormone levels. Forty-eight young adult females monitored their menstrual cycles for three replications and then reported for testing on Day 2, 8, or 14 of their cycles or on proportionally equivalent days for cycles longer or shorter than 28 days. Results agreed precisely with those of Wells and Payne in showing significantly higher reminiscence on Day 14 (high estrogen) than on the other days (low estrogen). This effect was attributed to larger accumulations of reactive inhibition (Hull, 1943) by high-estrogen subjects, evidence for which was found in the shallower slopes of their prerest performance curves. An estrogen-activation hypothesis was elaborated to account for the higher levels of reactive inhibition.
\end{abstract}

Several studies of psychomotor learning and performance, conducted in this laboratory and elsewhere, have shown that young adult females reminisce significantly more than do males of comparable age (Buxton \& Grant, 1939; Hsu \& Payne, 1979; Huang \& Payne, 1975; Payne \& Huang, 1977). This finding has appeared with monotonous regularity in rotary pursuit and mirrortracking tasks regardless of whether a conventional reminiscence paradigm or a paradigm involving alternating periods of work and rest is used as in distributed practice experiments (McBride \& Payne, in press; McCaffrey \& Payne, 1977).

We have found it useful to conceptualize this female dominance in terms of Hull's (1943) theory of reminiscence and distributed practice effects. According to this theory, the work involved in responding generates a negative motivational potential, reactive inhibition $\left(I_{R}\right)$, which accumulates as some function of the frequency and effortfulness of responding and subsides spontaneously as an exponential function of the amount of rest. As negative drive, $I_{R}$ is thought to oppose the excitatory tendency (E) arising from the interaction of learning (H) and drive (D), and this subtractive process leaves a residual excitatory tendency $(\overline{\mathrm{E}})$ that is presumably indexed by measures of performance. When rest occurs, $I_{R}$ subsides and $\bar{E}$ rises to yield the gain observed as reminiscence. Within this theoretical context, female dominance in reminiscence can be explained by supposing that females generate more $I_{R}$ per unit of prerest practice than males do and, having accumulated more $I_{R}$, they release more across rest in accordance with the specified exponential character of the subsidence function. Should this conceptualization be valid, one must find that (1) when the amount of prerest practice is held constant and the amount of rest is varied, females will reach a higher asymptotic level of reminiscence than males will, and (2) females will gain less proficiency per unit of prerest practice than males will because of their greater accumulation of $I_{R}$. Both expectations have been confirmed (McBride \& Payne, 1979).

What remains to be explained, of course, is the greater female accumulation of $I_{R}$ in prerest practice. Mindful of evidence for energizing properties of estrogenic hormones (Broverman, Klaiber, Kobayashi, \& Vogel, 1968; Logothetis, Harner, Morrell, \& Torres, 1959; Vogel, Broverman, \& Klaiber, 1971), Wells and Payne (1979) hypothesized that females exposed to massed practice when at or near the peaks of their estrogen cycles should generate more $I_{R}$, and hence should reminisce more, than when exposed at or near the nadirs of their cycles. Presumably, this would occur because high estrogen levels would increase the effortfulness of responding. Accordingly, they monitored the menstrual cycles of 43 young adults for two replications and then assigned the subjects without bias to testing sessions conducted on Days 2 (low estrogen), 8 (low estrogen), or 14 (high estrogen), or on proportionally equivalent days for subjects with cycles longer or shorter than 28 days. Despite this somewhat crude method of estimating the values of the independent variable, Wells and Payne found that subjects tested at or near their estrogenic peaks reminisced significantly more than did those tested on the nadir days. Mean reminiscence values at nadir were only slightly higher than those usually found in males. Since the average female has a more or less elevated estrogen level throughout approximately $70 \%$ of her menstrual cycle (Nocenti, 1968), the chances are about 7 in 10 of drawing a subject in that condition by sampling without regard to cycle phase, and this would suffice to impose a statistical bias in favor of greater female reminiscence.

The extraordinary character of the foregoing development suggested that any pursuit of the numerous implications arising from the estrogen activation hypothesis 
should be suspended until the repeatability of the Wells and Payne (1979) results could be ascertained. This was the objective of the present study.

\section{METHOD}

\section{Subjects}

The experimental sample consisted originally of 59 Caucasian students who volunteered to monitor their menstrual cycles for three consecutive replications and to report for testing on Day 2,8 , or 14 of the cycle, or on proportionally equivalent days for cycles longer or shorter than 28 days. Of the original 59 subjects, 48 fulfilled their testing commitments. These were distributed as follows: Day 2, $\mathrm{n}=15$; Day 8, $\mathrm{n}=16$; and Day 14, $\mathrm{n}=17$. Ages ranged from 17 to 22 years, with an average of 18.9 years. All subjects were right-handed and normally sighted. None acknowledged the use of contraceptive pills, and all reported themselves drug free on the day of testing.

\section{Task}

As in the previous study, this study employed a mirrortracking task that required subjects to use mirror vision to track a small silver target as it moved clockwise at $1 \mathrm{rpm}$ through a narrow star-shaped pathway. Subjects were instructed to keep the stylus on target as long as possible and to avoid contact with the sides of the pathway. Time on target was recorded in units of $.01 \mathrm{sec}$ for each $1 \mathrm{~min}$ of practice.

\section{Procedure}

The original 59 volunteers were assigned without bias to three groups defined in terms of testing day (i.e., Day 2, 8, or 14 of the menstrual cycle, or their proportional equivalents). Each of the 48 subjects who fulfilled their testing commitments notified the first author on Day 1 of her cycle and then reported for testing on the day to which she had been assigned. Following task instructions, all subjects were exposed to a practice sequence consisting of $3 \mathrm{~min}$ of massed practice (Trials 1-3), $3 \mathrm{~min}$ of rest, $3 \mathrm{~min}$ of massed practice (Trials 4-6), $3 \mathrm{~min}$ of rest, and $3 \mathrm{~min}$ of massed practice (Trials 7-9), as in Huang and Payne (1975) and Wells and Payne (1979). Debriefing concluded the testing session.

\section{RESULTS}

Experimental effects were measured in terms of (1) reminiscence scores and (2) performance scores achieved throughout the nine trials.

\section{Reminiscence}

The interpolation of two rest periods in the practice sequence permitted the computation of two reminiscence scores for each subject. The first was found by subtracting the score on Trial 3 from the score on Trial 4; the second was found by subtracting the score on Trial 6 from that on Trial 7. The 96 values were then subjected to a mixed-model variance analysis in which groups constituted the main effect between subjects and reminiscence periods provided the main effect within subjects. Neither period effects $[F(1,45)=2.99$, $\mathrm{p}>.05]$ nor the interaction of period and group effects $[\mathrm{F}(2,45)=1.88, \mathrm{p}>.05]$ was significant. Accordingly, a standard deviation of expected replication differences was derived from the between-subjects error term for use in directional $t$ tests of group mean differences. Inasmuch as the activation hypothesis specified that high
Table 1

Mean Reminiscence Values from Two Experiments

\begin{tabular}{cccc}
\hline & \multicolumn{3}{c}{ Test Day } \\
\cline { 2 - 4 } Experiments & 2 & 8 & 14 \\
\hline Wells \& Payne (1979) & 5.90 & 4.43 & 7.97 \\
Present Study & 4.73 & 3.99 & 7.60 \\
\hline
\end{tabular}

reminiscence scores should be found associated with high estrogen levels and vice versa, a comparison was made between the mean reminiscence of the Day 14 group and an equally weighted linear combination of the means of the Day 2 and Day 8 groups. The means thus compared were 7.6 and 4.36 , respectively $[t(45)=2.3$, $\mathrm{p}<.025]$. The null hypothesis was therefore rejected in favor of the activation hypothesis, as in Wells and Payne (1979).

The similarity of the present results to those reported earlier was even more striking than the foregoing analysis indicated. As shown in Table 1, the mean difference between the two studies was remarkably small on each testing day. Moreover, a variance analysis of combined data, in which groups and experiments constituted the main effects, showed a significant groups effect $[\mathrm{F}(2,85)$ $=5.42, \mathrm{p}<.025]$, but no significant experiments effect $(F<1)$ and no significant interaction of groups and experiments effects $(F<1)$. And, as Table 1 suggests, the mean of the Day 14 group was significantly higher than an equally weighted linear combination of the means of the two nadir groups $[t(85)=3.04, p<.001]$. Thus, the repeatability of the Wells and Payne (1979) results can no longer be seriously questioned.

\section{Performance}

As in the McBride and Payne (1979) study, the applicability of Hull's (1943) theory to the reminiscence data of the present study required evidence for a suppressive effect of $I_{R}$ upon prerest performance scores. If the higher mean reminiscence of the Day 14 group is to be attributed to a greater accumulation of $I_{R}$, one must find that the slopes of the Day 14 prerest performance curves are less steep than those of the two nadir groups. Accordingly, the 432 performance measures (48 subjects $X 9$ trials) were subjected to a mixed-model variance analysis in which groups constituted the main between-subjects effect and trial blocks and trials within blocks constituted the main within-subjects effects. As expected on grounds unrelated to the theory, the blocks effect and the trials effect were both significant $[F(2,90)$ $=144.11, \mathrm{p}<.001$, and $\mathrm{F}(2,90)=5.52, \mathrm{p}<.01$, respectively], and the overall groups effect was not significant $[F(2,45)=1.83, p>.05]$. Of crucial importance to the theory, however, was the significant interaction of groups and trials effects $[F(4,90)=3.33, p<.025]$. Since there were no other significant sources of variation, the test for interaction can be interpreted as a test for group trends across trials within blocks, and, as such, it shows that the group slopes were different. 


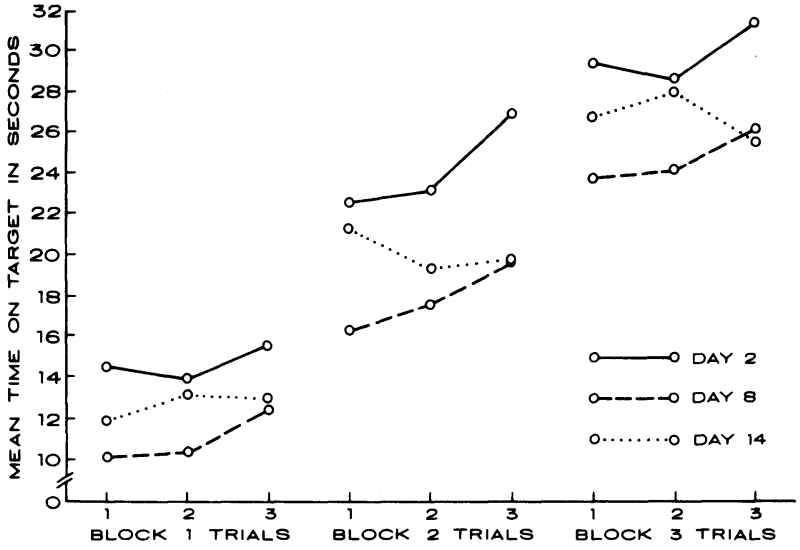

Figure 1. Day-group performance trends.

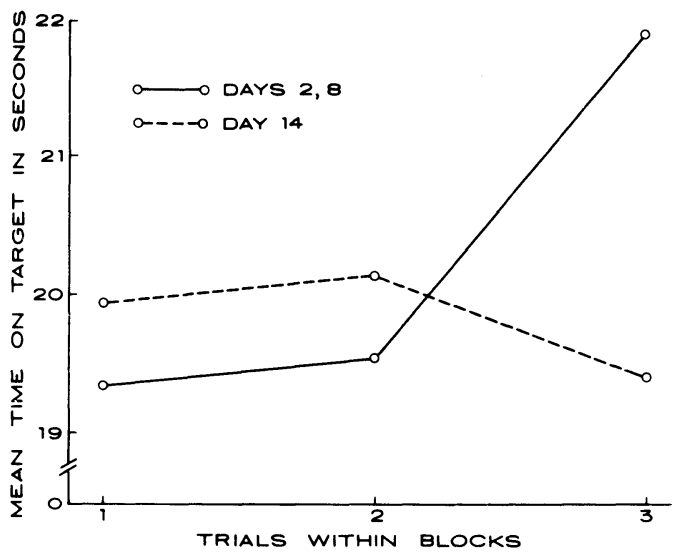

Figure 2. Performance trends of high-estrogen (Day 14) and low-estrogen (Days 2 and 8) groups after collapsing trials across blocks.

The nature of the group trend differences can be seen clearly in Figure 1. Here the slopes of the two nadir groups across trials within each block were generally positive, whereas the slopes of the Day 14 group were either less positive, as in Block 1 , or clearly negative, as in Blocks 2 and 3. When the two nadir groups were combined and the trial scores were collapsed across blocks, as in Figure 2, it is immediately apparent that the nadir condition was characterized by gain from Trial 1 to Trial $3[\mathrm{t}(90)=4.3, \mathrm{p}<.001]$, whereas the highestrogen condition was characterized not only by an absence of gain, but by some loss as well. It is reasonable to conclude, therefore, that the slope differences were concordant with theoretical requirements.

\section{DISCUSSION}

The two studies of the relationship between psychomotor reminiscence and phases of the menstrual cycle have now shown that subjects tested at times concurrent with the peaks of their estrogen cycles reminisced significantly more than those tested at times concurrent with the nadirs of their cycles. The studies offer no certainty, of course, that changes in the levels of the estrogenic hormones are responsible for the observed changes in reminiscence, for one must consider the fact that titers of other hormones (e.g., the gonadotropins FSH and LH) are also low during the early part of the cycle and high at ovulation. There may also be other correlated physiological events that have more explanatory value. For the present, however, we focus on the estrogens for two reasons. First, unlike other hormones involved in the menstrual cycle, the titers of estrogenic hormones are more or less elevated throughout approximately $70 \%$ of the average cycle (Nocenti, 1968), and, as noted earlier, this suggests a plausible explanation of the sex difference in reminiscence when females are sampled without regard for the cycle phase in which they happen to be. Second, numerous studies involving humans and lower animals have shown that increased levels of the estrogens are associated with increased levels of response activation (e.g., Broverman et al., 1968; Gerall, Napoli, \& Cooper, 1973; Kawakami \& Sawyer, 1967; Vogel et al., 1971). We shall return to this theme following a comment on another theoretical aspect of the reminiscence data.

The results of the present study, those of its precursor (Wells \& Payne, 1979), and those concerned with sex differences in reminiscence (Huang \& Payne, 1975; McBride \& Payne, 1979) all lend themselves readily to interpretation in terms of $I_{R}$ theory (Hull, 1943). If reminiscence is due to the exponential subsidence of $I_{R}$ across rest, as Hull argued, it follows that differences in the amount of reminiscence are due to differences in the amount of $I_{R}$ accumulated prior to rest. Because of the postulated suppressive effect of $I_{R}$ upon performance, such differences, in turn, should be related inversely to the rate of gain found in prerest practice. Thus, higher reminiscence values should be found associated with lower gains per unit of prerest practice. Within this theoretical context, therefore, one can attribute the high female reminiscence at ovulation to the accumulation of higher levels of prerest inhibition, evidence for which was in fact found in the smaller slopes of their prerest practice curves. When male and female reminiscence tendencies are compared, as in Huang and Payne (1975) and McBride and Payne (1979), one can likewise attribute the higher female reminiscence to higher levels of prerest inhibition, evidence for which was in fact found in the smaller slopes of the prerest performance curves.

The crucial theoretical problem, however, is that of rationalizing the higher accumulation of prerest inhibition. Why should females accumulate more prerest $\mathbf{I}_{\mathbf{R}}$ than males do, and why should ovulatory females accumulate more prerest $I_{R}$ than do females at the nadirs of their estrogen cycles? Wells and Payne (1979) proposed an estrogen activation hypothesis that said, in effect, that higher levels of the estrogens increase the intensity of responding, a condition that, in Hull's (1943) theory, should result in the generation and accumulation of higher levels of $\mathbf{I}_{\mathbf{R}}$. Although this view appears consistent with the experimental literature on behavioral effects of estrogens, it offers no insight into the mechanism(s) that might be involved. According to an analysis by Vogel et al. (1971), estrogenic hormones may augment the actions of one or more neurotransmitters, such as epinephrine, norepinephrine, and serotonin, by inhibiting monoamine oxidase (MAO). This enhancement of central adrenergic activity might well account for an increased intensity of response activation, for it is well established that known MAO inhibitors, such as iproniazid, can function as potent stimulants to produce an increased sense of well-being and heightened psychomotor activity. It bears repeating that this consequence would fulfill one of Hull's specifications for the generation of $\mathrm{I}_{\mathbf{R}}$.

Appealing as the foregoing hypothesis may be, at least to us, it is obvious that much work remains before one can be confident that estrogenic hormones play a causal role in the reminiscence phenomena reported. Among the more obvious suggestions for further work are studies of (1) reminiscence tendencies in pre- and postmenopausal subjects, (2) sex differences in reminiscence among the aged, and (3) effects of contraceptive pills upon reminiscence across the phases of the menstrual cycle. These studies are now underway. 


\section{REFERENCES}

Broverman, D. M., Klaiber, E. L., Kobayashi, Y., \& Vogel, W. Roles of activation and inhibition in sex differences in cognitive abilities. Psychological Review, 1968, 75, 23-50.

Buxton, C. E., \& Grant, D. A. Retroaction and gains in motor learning: II. Sex differences, and a further analysis of gains. Journal of Experimental Psychology, 1939, 25, 198-208.

Gerall, A. A., Napoli, A. M., \& Cooper, U. C. Daily and hourly estrous running in intact, spayed and estrone implanted rats. Physiology \& Behavior, 1973, 10, 225-229.

Hsu, S. H., \& PAYNe, R. B. Effector localization and transfer of reactive inhibition. Journal of Motor Behavior, 1979, 11, 153-158.

Huang, K. L., \& Payne, R. B. Individual and sex differences in reminiscence. Memory \& Cognition, 1975, 3, 252-256.

Hull, C. L. Principles of behavior. New York: Appleton-CenturyCrofts, 1943.

KawaKami, M., \& Sawyer, C. Effects of hormones and antifertility steroids on brain thresholds in the rabbit. Endocrinology, $1967,80,857-871$.

Logothetis, J., Harner, R., Morrell, F., \& Torres, F. The role of estrogens in catamenial exacerbation of epilepsy. Neurology, 1959, 9, 352-360.
McBride, D. K., \& PAyne, R. B. Psychomotor reminiscence as a function of sex and length of rest period. Journal of Motor Behavior, 1979, 11, 59-64.

McBride, D. K., \& PAYNe, R. B. The sex difference in rotary pursuit performance: Aptitude or inhibition? Journal of Motor Behavior, in press.

MCCAfFrey, R. J., \& PAyne, R. B. Interaction of sex and practice distribution effects. Bulletin of the Psychonomic Society, 1977, 10, 382-384

Nocenti, M. R. Reproduction. In V. B. Mountcastle (Ed.), Medical physiology. St. Louis: Mosby, 1968.

Payne, R. B., \& HuANG, K. L. Interaction of sex and task differences in reminiscence. Journal of Motor Behavior, 1977, 9 , 29-32.

Vogel, W., Broverman, D. M., \& Klaiber, E. L. EeG responses in regularly menstruating women and in amenorrheic women treated with ovarian hormones. Science, 1971, 172 , 388-391.

Wells, K. C., \& Payne, R. B. Psychomotor reminiscence as a function of gonadal steroid hormone variation. Bulletin of the Psychonomic Society, 1979, 14, 197-200.

(Received for publication January 30, 1981.) 